anatomical template for ingrowth of the host's tissues. The process of freeze-drying apparently alters the nucleic acids in the homografts, reducing their capacity to evoke immunological responses. Commander Hyatt also discussed the ethics and philosophy underlying this enterprise.

Prof. C. G. Rob said that in his clinical experience the general health of the patient and state of his other arteries are the most important factors governing a successful outcome of homografting frozen or freeze-dried arteries. Mr. H. A. Sissons finds that freeze-dried bone sterilized by irradiation has advantages over homografts preserved by other means. Grafting the cornea presents unique possibilities and problems. Because the tissue is avascular, immunological reactions to homografts do not occur, but viability was apparently essential if grafts were to retain transparency. Col. F. Hénaff has, however, obtained striking results with freeze-dried corneal homo- and hetero-grafts in rabbit, dog and man. Lamellar grafts invariably took, and some of the full thickness homografts also remained transparent although the tissue was undoubtedly dead.

By contrast, pathogenic protozoa, spermatozoa and endocrine tissue can never perform their specific functions unless they are alive. They can be preserved for long periods in the frozen state in presence of glycerol at low temperatures, but cannot be stored after freeze-drying from this medium because of the lethal effects of concentrated glycerol at room temperature. Dr. C. Polge and Dr. M. A. Soltys reported that, in presence of $7 \cdot 5-10$ per cent of xylose, glucose or sucrose, and in absence of salts, bull spermatozoa suspended in egg yolk and trypanosomes in 10 per cent horse serum survived freezing at temperatures down to $-40^{\circ} \mathrm{C}$. In these media they might survive freeze-drying. Dr. Parkes envisaged the protection of endocrine tissues during freezing by substances which would volatilize together with ice during high-vacuum distillation. Methanol is sufficiently volatile at low temperatures, and rat ovaries have survived freezing for several hours in its presence. Future developments along these lines may eventually permit long-term storage of mammalian cells without loss of viability in the dry state at room temperatures.

The interest of the meetings was unquestionable and the proceedings, to be edited by Dr. A. S. Parkes and published by Blackwell Scientific Publications, Ltd., should provide a basis for further advances in this branch of natural philosophy. The secretary of the Symposium, Dr. R. J. C. Harris, and the Institute of Biology, are again to be congratulated on their initiative.

AUdRey U. SMITH

${ }^{1}$ Smith, A. U., in "Biological Applications of Freezing and Drying", ed. by R. J. C. Harris (Academic Press, Inc., New York, 1954) Biol. Rev., 33, 197 (1958)

${ }^{2}$ Luyet, B. J., and Gehenio, P. M., "Life and Death at Low Temperatures" (Biodynamica, Normandy, Miss., 1940); in "Temperature : Its Measurement and Control in Science and Industry" (Reinhold Publishing Corp., New York, 1941); Colloid Chem., 5 , 859 (1944).

s Altmann, R., "Die Elementarorganismen und ihre Beziehungen zu den Zèlen" (Veit and Co., Leipzig, 1890).

Shackell, L. F, , Amer. J. Physiol., 24, 325 (1909).

"Wollaston, W. H., Phil. Trans. Roy. Soc., 108, 71 (1813).

"Harris, R. J. C. "Freezing and Drying" (Institute of Biology, London, 1951); Nature, 168, 851 (1951).

' Lovelock, J. E., Nature, 173, 659 (1954).

Andjus, R. K., and Smith, A. U., J. Physiol., 128, 446 (1955); Smith, A. U., Lovelock, J. E., and Parkes, A. S., Nature, 178,1136 (1954).

\title{
NOISE IN INDUSTRY
}

$\mathrm{N}$ OISE in industry was the subject of the eighth conference of the British Occupational Hygiene Society, which was held in London on April 1. The proceedings opened with a paper on the clinical and physiological effects of noise by Mr. T. Cawthorne (King's College Hospital, London), who said that hearing was the last of the special senses developed in animals in the course of evolution. The reception apparatus arose as an outgrowth of the organ of balance, and disorders of hearing were accompanied by disorders of balance. Hearing was primarily a protective device which operated through the central connexions of the eighth nerve, some of which went to the temporal lobe, where sound was appreciated, and some directly to motor nerves to produce a sound reflex action even before the animal was conscious of the sound it reacted to. Propagation of the species was another function which was often aided by hearing, in man as well as in the lower orders, but man was unique in his possession of speech and in his social activities, which depended very much upon unimpaired hearing. Deaf persons were under a constant stress on this account.

Sound waves in air passed down the ear canal and generated vibrations of the ear drum which were transmitted across the air-filled middle ear by a chain of ossicles. The innermost of the ossicles rocked in the oval window of the inner ear, or cochlea, and caused oscillations to pass through the fluid which filled it. The fluid waves caused the basilar membrane of the cochlea to spring up and down. This membrane carried the organ of Corti, which converted the move- ments to tactile stimuli of nerves. The organ carried hair cells which were brought into contact with a leaf-like structure above them when the basilar membrane moved. Excessive movement strained the hair cells and acoustic trauma took place mainly, if not entirely, in the organ of Corti.

Early deafness resulting from exposure to noise very often produced a loss in sensitivity to frequencies in the vicinity of 4,000 cycles. This was found in men employed in metal stamping and chipping, and in those who shot two or three thousand cartridges a year.

Both the intensity of noise and its duration were significant in causing trauma. Regular exposure over long periods to more than 85-90 decibels resulted in irreversible changes taking place in the organ of hearing. Individual sensitivity varied and unduly sensitive entrants to industry should be picked out by tests. One way of doing this was the estimation of post-stimulatory fatigue.

High-tone deafness made it difficult to interpret speech, particularly when there was a noisy background; good binaural hearing was an asset in the latter situation. High-tone deafness caused by industrial trauma resulted in a time lag becoming apparent in the apprehension of speech. The importance of speaking slowly when addressing an audience arises from this effect, which might also be due to fatigue on the part of the listeners.

The encouragement of workers to protect themselves against excessive noise called for propaganda. There was little hesitation in guarding against an obvious 
risk of injury, but the threat of deafness was apt to be disregarded because it would be $10-15$ years before it happened. Protective devices were available which gave 15-30 decibels reduction. This sufficed for most industrial noises but was inadequate for really tremendous sounds such as those issuing from jet engines.

Speaking on noise measurement, the analysis and evaluation of harmful effects, Dr. T. S. Littler (Wernher Research Unit on Deafness), stated that the ear was a pressure-sensitive mechanism. Normal atmospheric pressure was about one million dynes per square centimetre; during production of a very intense sound a variation of the order of 2,000 dynes per square centimetre was superimposed. Such a sound was generated in the vicinity of a jet engine and had a level of 140 decibels. Full specification of a sound involved giving its waveform, but in dealing with noise it often sufficed to know the root mean square value of the pressure-level, averaged over a short interval, and the peak pressure attained during the same time.

The waveform could be analysed by the Fourier method; in practice, an approximation to this was achieved by selecting limited frequency ranges by means of filters, which could be made to accept frequencies over bands ranging from a few cycles to an octave. Component pressures measured over these ranges were not additive; they had to be converted to intensities first. The resultant intensity could then be reconverted into pressure if desired.

The human ear did not behave like a selective filter because a pure tone at a given frequency excited a considerable region of the analytical mechanism of the cochlea; the selective capacity of the ear depended both on the cochlea and on the neural pathway. It was found that an intense pure tone produced most auditory fatigue in regions of the ear specific to a frequency about half an octave higher than that of the fatiguing tone.

A pure tone was masked by those components of noise which were situated in a band of frequencies centred on that of the tone. This was called the critical band and increased in width from 25 cycles to 200 cycles in ascending through the audible range. It was supposed that the critical band region of frequency in a noise was the range responsible for damage to the ear in a particular frequency range. Another body of opinion accepted it as sufficient to analyse noise into octave bands for the investigation of noise hazards; such an analysis was inadequate for the determination of critical band-levels unless the noise level was fairly uniform throughout the octave bands. Since we were uncertain of the physical basis of noise trauma, narrow-band analysis was always a useful adjunct to octave or part-octave analysis. It helped to detect the sharply rising waveform, which produced a characteristic group of harmonics and was regarded by some as being highly traumatic.

In order to define impairment of hearing a standard of normality was required. That adopted in the United States corresponded to sound pressure-levels which were 10-15 decibels higher than the British; the British assessment of loss of hearing was therefore more stringent. Since both the United States and the British standards specified acoustical pressures at the meatus, it was possible to convert observations expressed on one basis to the other system.

Audiograms of people exposed to noise often showed a drop in response round about 4,000 c./s. which was known as the traumatic dip or notch. It was possible that this loss was associated with percussive noises and there was an indication that physical and physiological factors combined to make the ear specially sensitive at frequencies near $3,500 \mathrm{c} . / \mathrm{s}$. and render it more easily damaged.

Protection of the ears was afforded by the insertion of plugs or the application of external muffs. When an ear plug blocked the meatus the cartilaginous surround provided the main path of entry for sound. If the auricle was covered by an efficient ear muff the impact of sound on the most permeable region was much reduced. It was essential that the muff should not be porous and the most efficient type embodied a liquid-filled plastic pad. A good seal against the contours of the bony area around the auricle could be obtained by light pressure.

With such a protector in place only bone conduction remained as a route of ingress. Since this was estimated as being 50-60 decibels below other forms of entry it was not an important factor in protecting against harmful noise.

In any programme of hearing conservation it was necessary for an otological examination and an audiometric test to precede exposure to noise. There were differences of individual susceptibility to be allowed for; it was not possible to say whether someone already deaf would be less vulnerable to a given noise than a normal person.

Mr. N. Fleming (National Physical Laboratory), joint author with Mr. W. C. Copeland, read a paper on the principles of noise suppression. Explaining the physical description of noise in terms of decibel levels of sound pressure and frequency-band analysis, he said it is necessary to supplement these concepts with a subjective indication of loudness which is provided by the phon scale. This is based on the sensation of loudness produced by a pure tone of $1,000 \mathrm{c} . / \mathrm{s}$.; the loudness-level in phons is defined as being numerically equal to its sound pressure-level in decibels. The loudness-level of any other noise is then gauged by comparison with the pure tone, which is adjusted until it appears to have the same loudness as the noise when heard under prescribed conditions. Owing to this arbitrary definition there is no obvious relation between the numbers of the scale of phons and the corresponding sensations.

To overcome this disadvantage another scale has been devised in which the scale number is intended to be directly proportional to the sensation of loudness. The unit on this scale is called the sone. It has been agreed internationally that a loudness of one sone is equal to 40 phons and that 10 phons are required to double the sensation of loudness. Thus a loudness of 8 sones is equal to 70 phons and the pressure-level of a pure 1,000 cycle tone of the same loudness would be 70 decibels. The absolute pressure, $p$ dynes $/ \mathrm{cm}^{2}$, would be given by $70=20 \log _{10}(p / 0 \cdot 0002)$. This degree of loudness corresponds to the sound produced in a city office with the windows open or to conversational speech.

Subjective measurements of loudness have to be made with a group of observers, but it is possible to get some idea of loudness from octave-band analysis of noise.

The remainder of the paper dealt with the suppression of noise and was concerned, first, with planning to avoid noise. Noisy production operations in a factory should be segregated so that there is no needless exposure of people engaged on quieter work. Escape routes for noise, such as access doors, open roof lights and thin roofs, have to be considered. The 
nuisance caused by noise at a distance from a factory may be due to sources which are not apparent inside or in its immediate environment.

Handling noises can often be much reduced by using plastic- or rubber-covered benches and bins; delivery chutes should be smooth inside and designed to avoid too great a distance of fall into bins.

Sound insulation or absorption is employed when a noisy source cannot be eliminated. An insulating barrier may absorb or reflect sound. The process of absorption involves the dissipation of sound energy as heat and takes place either by the barrier itself vibrating or by the vibrating air inside cavities in porous insulation wasting its energy as friction.

The sound insulation of a partition is expressed by its sound reduction index, which is the difference between the sound pressure-levels on the incident and transmitted sides. There is a good correlation between the index and the weight per square foot for homogeneous sheet materials. Best results are obtained with isolated panels separated by an air gap. In general, high-pitched sounds are likely to have a greater sound reduction index than lowpitched ones, for which weight of insulator is the predominating factor. Irregularities of transmission are more common at higher frequencies.

If a machine is totally enclosed in reflective insulation the sound-level inside will build up. Absorption is necessary as well. The best absorbers are porous materials, such as mineral wool; though thin layers are effective at high frequencies, large thicknesses become necessary at low. When a very high degree of sound insulation is required small leaks through a partition must be avoided. A sound-reduction index of 40 decibels means that only 1 part in $10^{4}$ of the incident sound energy is transmitted. An aperture with an area of only $1 / 10,000$ of that of the insulated partition will let through as much sound as the entire partition. When apertures have to be made they can be provided with sound-attenuating ducts, which are also of value in ventilating systems to eliminate fan noise. The principle of such ducts is absorption at the wall, and their efficiency is increased by subdivision into a number of narrow channels. Noise reduction in decibels is proportional to the length. Attenuation increases with frequency up to a point at which the wave length of the sound is equal to the channel width.

Anti-vibration mountings are of value in reducing noise transmitted from machines; they should be designed so that the natural frequency of the machine on its resilient supports is much lower then that of the objectionable vibration. Complaints are sometimes aroused by low-frequency pulsation, as from engine exhausts. The fitting of an expansion chamber about
15 times the volume swept by the piston at each stroke and an absorbent wall silencer had been helpful in such cases.

The final paper, read by Dr. Alan Carpenter (Medical Research Council Applied Psychology Research Unit, Cambridge), deslt with the question whether noise which was not loud enough either to produce actual physical damage or to arouse annoyance could yet be responsible for interference with mental work.

An experiment performed a quarter of a century ago indicated that the output of work from weavers was increased very slightly when they wore ear plugs which reduced the noise transmitted by 10-15 decibels, in spite of the fact that the workers did not like using the ear plugs and thought they were of no value. More than fifty years ago it had been demonstrated that a slowing down of performance was produced every time an intermittent noise was turned on or off, with a return to a normal speed of working between times.

Noise which had meaning was more distracting than meaningless sound, especially if the meaning was a little elusive. It was probable, too, that noise was more disturbing to a worker engaged on a task with which he was not very familiar.

Recent work had resulted in the formulation of the hypothesis of the 'mental blink', which had some analogy with eye blinking. Experiments suggested that effective regulating mechanisms existed in man which tended to maintsin his output quality in spite of disturbing influences. They were analogous to feed-back in physical servo systems or to the maintenance of arterial blood pressure and other physiological devices. When such a mechanism operated it was unprofitable to look for changes of output resulting from external stimuli ; it was better to examine the signal process or to seek for some aspect of the behaviour of the system outside its regulation. The 'mental blink', or a shifting away and shifting back of attention upon a mental task, provided such an opportunity. A subject in a good state of motivation would bring his attention back to a monotonous task in one and a half to two seconds. Attention could not be constantly held but tended to wander.

It was probable that in a watch-keeping job, where slackening of attention could result in an expensive mishap, a strong case existed for excluding loud noise. In inspection, too, where lapses of vigilance would increase the number of incorrect articles to be accepted, loud noises should be eschewed.

A full account of the Conference will be published in the first issue of the Society's journal, Annals of Occupational Hygiene.

C. N. Davies

\section{OBITUARIES}

\section{Dr. C. E. P. Brooks, I.S.O.}

W'rTH the death of Dr. C. E. P. Brooks on December 14, climatology in Britain lost its most eminent authority and the meteorological profession one of its most versatile members.

Dr. Brooks was born in London on November 10 , 1888. He was educated at University College School, London, and entered the Meteorological Office as a probationer in 1907. Studying in his own time, he graduated B.Sc. (London) with honours in geology in
1912. He proceeded to M.Sc. in 1916, and in 1926 the D.Se. was awarded to him for a thesis on "The Variations of Pressure in the Neighbourhood of the British Isles".

$\mathrm{He}$ served in the Meteorological Office for more than forty years, retiring in 1948 as assistant director (climatology), though he continued part-time work for the Office, mainly on the application of statistical methods in meteorology, for a further two years. Shortly before retiring, he was made a Companion of the Imperial Service Order. 